\title{
Production of strawberry using internet of things: a review
}

\author{
Olakunle Elijah, Abdelmoneim A. Bakhit, Tharek A. Rahman, Tien Han Chua, \\ Siti Fatimah Ausordin, Rifhan Narrissa Razali \\ Wireless Communication Center, School of Electrical Engineering, Universiti Teknologi Malaysia
}

\begin{tabular}{|c|c|}
\hline Article Info & ABSTRACT \\
\hline $\begin{array}{l}\text { Article history: } \\
\text { Received Nov 13, } 2018 \\
\text { Revised Jan 9, 2019 } \\
\text { Accepted Jan 25, } 2019 \\
\text { Keywords: } \\
\text { Greenhouse } \\
\text { Internet of things } \\
\text { Low power wide area (LPWA) } \\
\text { Smart agriculture } \\
\text { Strawberry }\end{array}$ & $\begin{array}{l}\text { Strawberry is a widely consumed fruit and cultivated globally but } \\
\text { predominantly in cooler temperate zones because of its wealth of nutrients, } \\
\text { vitamins and minerals. However, there are several challenging factors faced } \\
\text { in the growing of strawberry such as temperature, seasonal production and } \\
\text { short shelf-life. To overcome some of these challenges, the application of } \\
\text { greenhouse and internet of things (IoT) are being used. In this paper, a } \\
\text { review of the application of IoT in the greenhouse production of strawberry } \\
\text { is presented. This includes a survey of existing literature on production of } \\
\text { strawberry, types, method of growing strawberry and the application of IoT. } \\
\text { The benefits and challenges of IoT in greenhouse strawberry production are } \\
\text { discussed. The review highlights the need for use of low power wide area } \\
\text { communication technology in the application of IoT in greenhouse } \\
\text { strawberry production especially in tropical regions like Malaysia. }\end{array}$ \\
\hline & $\begin{array}{r}\text { Copyright }(2019 \text { Institute of Advanced Engineering and Science. } \\
\text { All rights reserved. }\end{array}$ \\
\hline \multicolumn{2}{|l|}{ Corresponding Author: } \\
\hline $\begin{array}{l}\text { Olakunle Elijah, } \\
\text { Wireless Communication Center, } \\
\text { School of Electrical Engineering, } \\
\text { Universiti Teknologi Malaysia. } \\
\text { Email: elij_olak@yahoo.com }\end{array}$ & \\
\hline
\end{tabular}

\section{INTRODUCTION}

The strawberry fruit is a widely consumed and cultivated globally but predominantly in cooler temperate zones because of its wealth of nutrients, vitamins and minerals. Strawberry belongs to the Rosaceae family, genu Fragaria and is a perennial plant which require pollination to bear fruits. The flowering is influenced by photo-period and temperature. There are several places that cultivate strawberry such as South Kashmir [1], Japan [2], Korea [3], Malaysia [4], and United States [5]. The United States is known to be the largest producer of strawberry. The cultivated varieties are classified into June-bearing [6], day-neutral [7] and ever-bearing cultivars [8]. Table 1 summarizes the difference between the strawberry cultivars. However, there are several challenging factors faced in the growing of strawberry such as temperature, seasonal production and short shelf life. To overcome these challenges, forced cultivation are employed. Some of the forced cultivation include the use of troughs, styrofoam in greenhouse and winter bags and pots in glasshouses. In this paper, we focus on the application of IoT in greenhouse production of strawberry especially in Malaysia. The Malaysian climate is categorised as equatorial which is hot and humid throughout the year with the average temperature of 27 degrees Celsius and while humidity is within the range of $60 \%-70 \%$. In Malaysia, strawberry is grown in the Cameron Highlands where there is low temperature and cooling climate. The use of greenhouse cultivation can make it possible to grow strawberry in other locations in Malaysia. 
Table 1. Summary of Strawberry Classifications

\begin{tabular}{cccc}
\hline & June-bearing & Day-neutral & Ever-bearing \\
\hline Production & Once per year & Throughout year-long growing seasons & Twice per year \\
Temperature & $<15{ }^{\circ} \mathrm{C}$ & $2-29{ }^{\circ} \mathrm{C}$ & $15-21{ }^{\circ} \mathrm{C}$ \\
Seasons & & Spring and late summer & Late June - early July, August \\
Life span & $3-5$ years & $2-3$ years & $3-4$ years \\
\hline
\end{tabular}

There are several methods of growing strawberry while certain climatic conditions are required to produce high quality fruits. The strawberries grow by producing stolons which spread out from the base plant to form new plants. It then undergoes flowering which needs to be pollinated by bees or other insects. The flowers develop into fruits. The strawberry plant is shown in Figure 1(a). Figure 1(b) illustrates the different methods of cultivating strawberry. Conventionally, the strawberry plants require warm days and cool nights and prefer well-drained sandy soil rich in organic matter to grow. The use of greenhouse is an alternative method to grow strawberries. A greenhouse is one of the methods that is common and widely used in modern agriculture. It is used when the plant needs certain environmental conditions to grow. Some of the significant factors for using a greenhouse is that it brings great economic income [9] with high quality yield and low cost [10]. Research has shown the acceptable climatic conditions required for the growth of strawberries as presented in Table 2. The temperature and humidity affect the size, colour and taste of sweetness of the fruit. The emergence of IoT and its application in agriculture allows for remote monitoring and automation, paving the way for smart agriculture. Several works have investigated the use of greenhouse in production of tomatoes, lettuces, strawberries, mushrooms and spinach [11-13]. The use of IoT in greenhouse production helps increase quality, avoids waste of resources, reduce physical effort and time with acceptable cost. The ability to simulate the environmental condition in the greenhouse allows for off-season production of the strawberry. The temperature affects the sweetness and size of the strawberry. For instance, according to [1], the optimum day time temperature of $22^{\circ} \mathrm{C}$ and night time temperature of $13{ }^{\circ} \mathrm{C}$ at night is required. The humidity required should be between $50 / \pm 10 \%$. The soil $\mathrm{pH}$ should be between 6.0 to 7.0 range. The sun light is important to complete the plant growth cycle for $16 / 8-\mathrm{h}$ light/dark (L/D) [8]. The variation of the parameters will affect the size, sweetness, taste and colour of the fruit.

From Figure 1(b), the strawberries can be grown either as seeds or as plants either outdoor (field) or indoors (Greenhouse). In the greenhouse, planters can be used or hydroponic or planted on the ground.

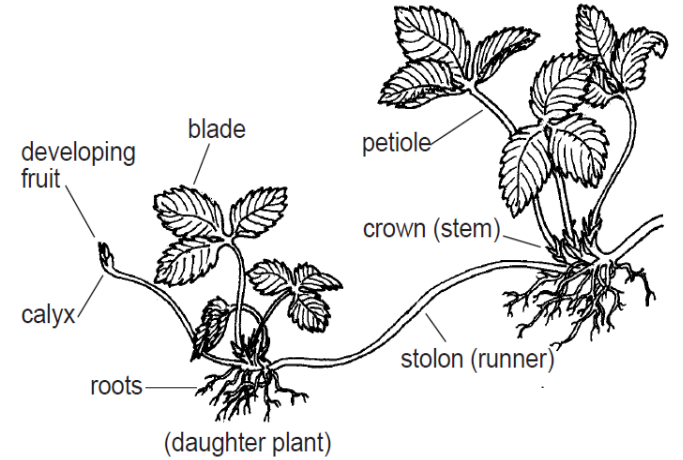

(a)

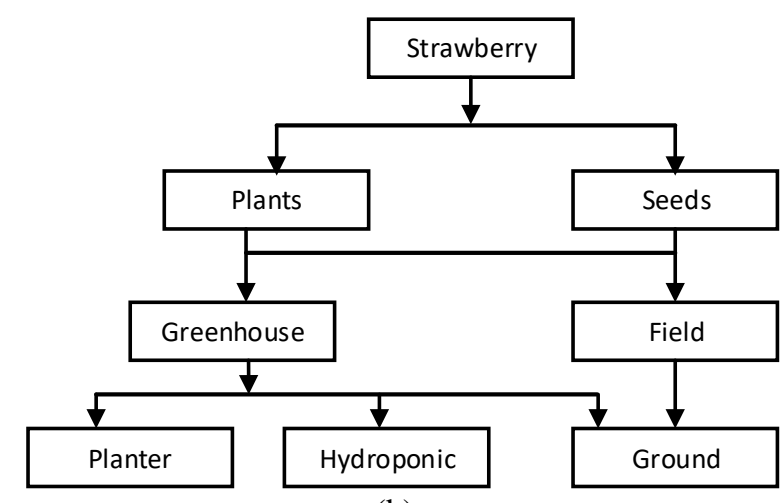

(b)

Figure 1. (a) Strawberry plant (b) Method of cultivating strawberry

Table 2. The Climatic Conditions to Grow Strawberry

\begin{tabular}{cc}
\hline Climatic conditions & Value \\
\hline Temperature & $22-13{ }^{\circ} \mathrm{C}$ \\
Humidity & $50 / \pm 10 \%$ \\
Soil pH & 6.0 to 7.0 \\
Sunlight & $16 / 8-\mathrm{h}$ \\
\hline
\end{tabular}




\section{LITERATURE REVIEW}

In this section, a review of related works on strawberry production using greenhouse and IoT technology is presented. The research on strawberry ranges from the study of the environmental conditions to the growth of strawberry [12,14, 15], smart harvesting methods [16-18], remote monitoring, and automated control of irrigation system [19]. Furthermore, this section covers the description of the IoT and its application to greenhouse production. The benefits and challenges of IoT for greenhouse strawberry production is also presented.

\subsection{Review of Related Work}

In [4], an automated temperature and humidity control system for greenhouse strawberry production using solar system for the Malaysian climate is presented. While the system is designed to monitor and automate the control of water moisture and temperature of the greenhouse, no fog misting was considered to control the humidity. The authors in [12], considered the light intensity and $\mathrm{CO} 2$ concentration in strawberries greenhouse. An intelligent system that monitors and controls the amount of $\mathrm{CO} 2$ and light intensity was developed. However, other parameters like the temperature, humidity and soil moisture are not covered. Further work carried out by [20-23] have considered the use of IoT with greenhouse. In [20], a data compressing protocol $(\mathrm{CP})$ is proposed for IoT based greenhouse monitoring. The use of the $\mathrm{CP}$ is to increase the efficiency of data transmission from sensor nodes to master controller in the network. In [21], a greenhouse sensor intelligent management system based on internet of things was implemented. A threelayered architecture that considers the sensing, network and application layer in greenhouse is presented. The air and soil temperature, air and soil humidity, soil $\mathrm{pH}$, moisture content and light intensity were monitored. The IoT communication is based on Zigbee and General Packet Radio Service (GPRS) communication technology. The authors in [22], studied the application of IoT on greenhouse production. The data such as temperature, humidity is monitored remotely and are sent via GPRS for management purposes. In [23], the use of IoT to monitor soil moisture, humidity, temperature, presence of fire, color of leaves and detection of toxic gases in greenhouse is presented. However, the IoT communication employs the use of GSM. The draw back to the works in [20-23] are high power consumption and short-range communication technology.

\subsection{Internet of Things}

The advancement in communication technology is allowing for devices to be controlled remotely at anytime and anywhere via the internet. IoT is considered as the network of devices, objects, things enabling the transfer of data without human interaction. IoT aims to make everything smart, intelligent and to solve the interconnection among thing-to-thing, human-to-thing, human-to-human, device-to-device and machineto-machine. This has made it possible for IoT to be used in several applications such as smart healthcare [24], smart parking [25], smart home [26], logistics management [27], agriculture [28, 29] and smart river monitoring system. IoT from electronic communication point of view is combination of physical things embedded with electronic circuits, sensors, and software connected by using the internet as the medium to communicate and exchange information while all the data are securely connected to the cloud [30]. The IoT consist of three major components which are sensor device, IoT cloud, and platform as shown in Figure 2. Earlier works on sensor deivce have made use of short-range communication devices such as Zigbee, 2G, and 3G network. The emergence of the low power wide area communication technologies for IoT devices promises low power consumption, long range communication, higher penetration power compared to $3 \mathrm{G}$, $4 \mathrm{G}$ cellular communication [31]. Some of the emerging LPWA communication standards are Narrow Band IoT (NB-IoT), LoRaWAN, enhanced MTC, Sigfox, weightless, Ingenu [32]. The LPWA offers $+20 \mathrm{~dB}$ gain over legacy cellular systems by using sub-1 GHz band, narrowband modulation, and spread spectrum techniques. In addition, LPWA offers ultra-low power operations via simplified topology of node devices. Examples are the star topology, application-based duty cycle for transmission of data, and light weight medium access control. The platform consists of the IoT cloud server and analytic server for data storage and analysis. The information from the IoT cloud can be accessed via mobile platforms and commands can be sent to the sensor devices via the IoT cloud.

\subsection{IoT in Greenhouse production of strawberry}

The set-up of IoT greenhouse production for strawberry suitable for Malaysia is presented in this section. The set-up consists of the greenhouse set-up, growing systems, irrigation system, automation system and monitoring system. First is the design of the greenhouse. The greenhouse can be made of plastic as shown in the Figure 3. The design takes into consideration the cooling system where ventilation fans are used for cool air circulation from the bottom to the top of the greenhouse. In addition, refrigeration cooling systems can be connected to underground ducting to allow for fresh air inlets. The use of thermal screen in the greenhouse is needed to prevent heat accumulation and to provide shade for the plants. Second is the 
structure for growing the strawberry. There are different methods of growing. Examples are use of polyethylene bag laid on the ground or placed on suspended Polyvinyl chloride (PVC) gutters, polygal troughs suspended in air and vertical systems where racks are mounted above each other. Third is the irrigation system. The irrigation system is an automated system that allows for water to be applied to the plants using drip tape or sprinklers. The irrigation system can also be used for supply of nutrients and organic pesticides. Fourth is the automation system. The use of IoT device help to automate the entire process in the greenhouse. This includes the control of irrigation system, humidity control (misting system), lighting system and cooling system. Sensors such as air temperature and humidity sensor, CO2 sensors, light sensors, pH sensors, rain detector are used to monitor the condition of the greenhouse. The data collected from these sensors are then used to automate the greenhouse. The fifth is the monitoring and control via IoT. The data acquired from the sensors can be transmitted to the IoT cloud via the LoRaWAN gateway. LoRa nodes can be used in connection with low power microcontrollers to transmit data to the IoT cloud. Examples of the IoT cloud are Thingspeak, The Things Network (TTN) and Carriots. The data from the IoT cloud are then analysed and compared with threshold required for action or control purpose. The data from the IoT cloud can also be monitored using a portable mobile device. The set-up of the greenhouse is illustrated in Figure 4 and a flowchart showing the control and automated system is shown in Figure 5. In Figure 4, the tanks are connected to the irrigation system using automatic valves and are placed in different levels of gravity to allow for easy flow and less numbers of pumping machine. The sensors in the greenhouse can be monitored using a pycom-fipy microcontroller which supports five communication modes. The communication modes include Wi-Fi, LoRa, Sigfox, Bluetooth and NB-IoT. The data acquired from the sensors are concatenated and sent by the pycom-fipy to the IoT cloud via the LoRaWAN. These data are stored and analysed in the cloud server. The use of internet allows these data to be viewed from mobile devices anywhere and anytime.

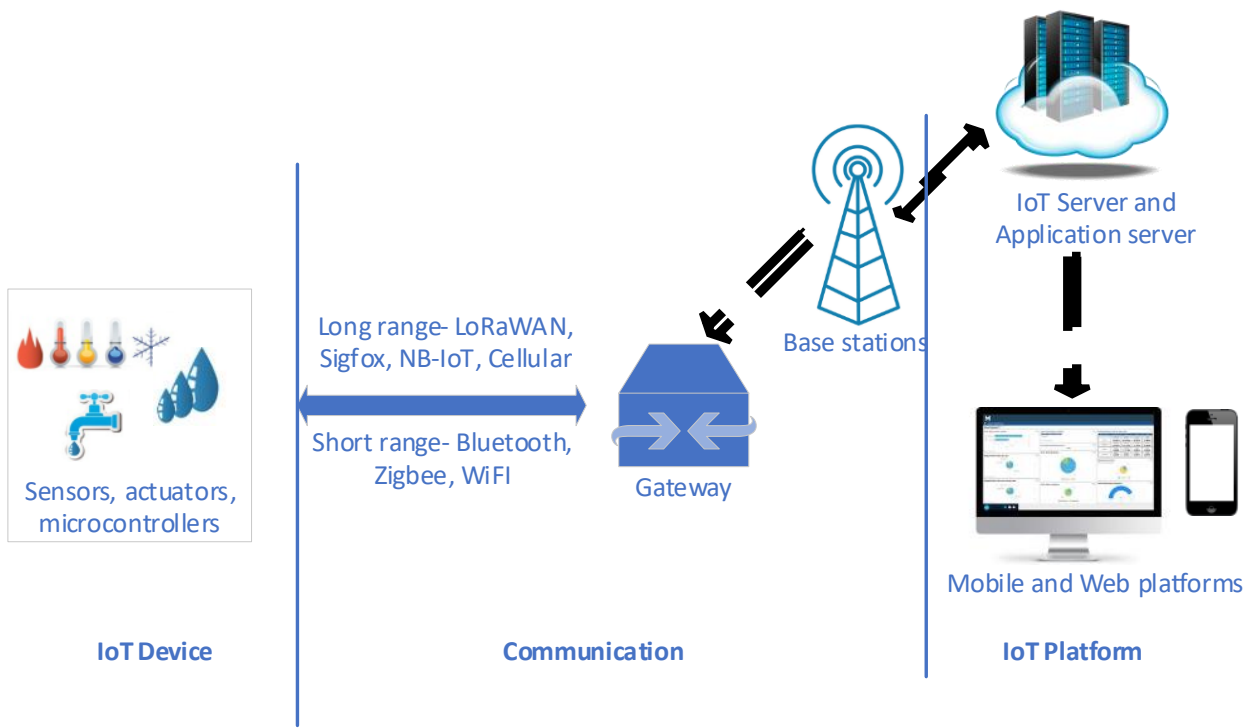

Figure 2. IoT architecture

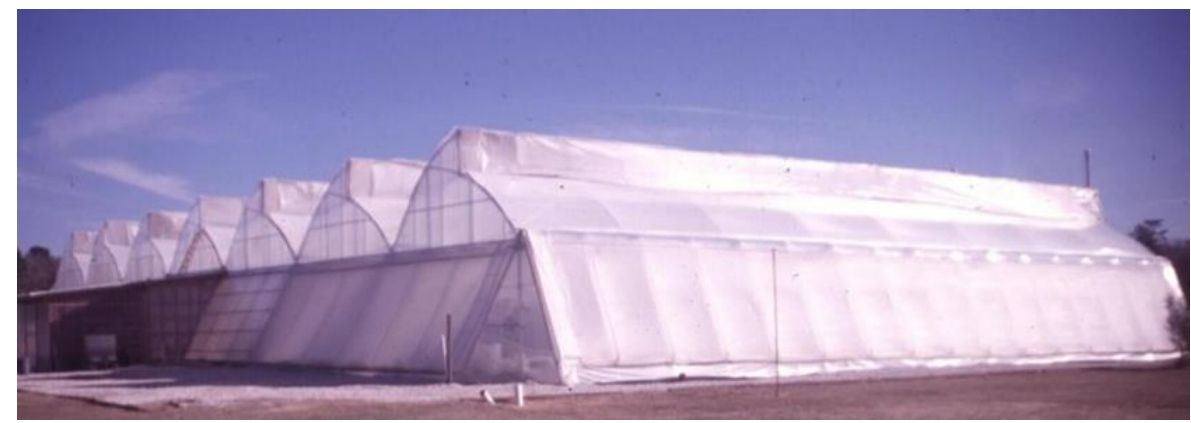

Figure 3. Typical plastic greenhouse 


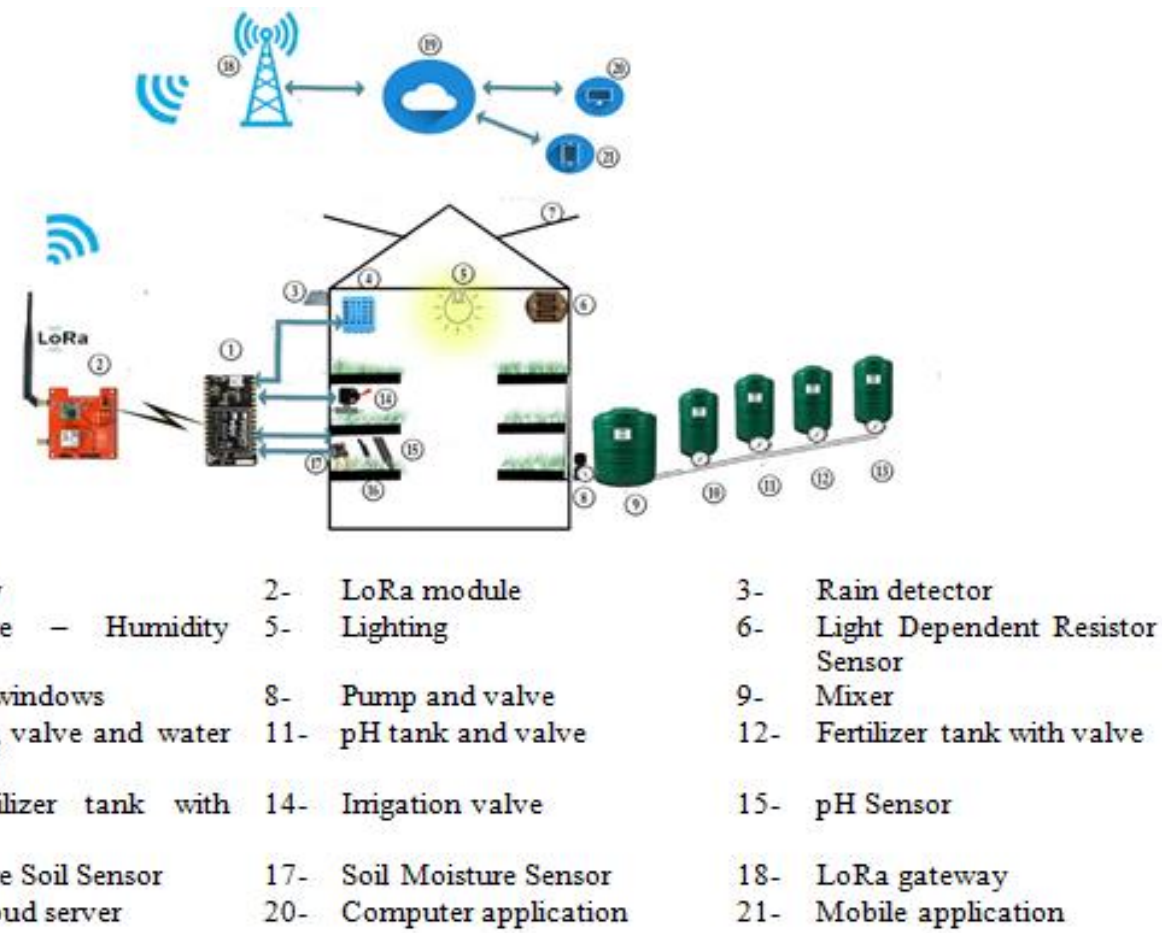

Figure 4. Typical greenhouse and remote control

\subsection{Benefits and Challenges}

There are several benefits that the IoT can provide in the greenhouse strawberry production. First, it helps to increase efficiency in terms of monitoring and data acquisition for research purposes. Furthermore, it will allow for precision application of nutrients, pesticide and water to the strawberry. Other advantages include the ability to track the delivery and supply of the strawberry by consumers using their mobile devices. It will also enable data profiling of the production of strawberry based on environmental condition, and the nutrient needed to optimise the yield of strawberry. However, there are challenges that can be associated with the use of IoT in greenhouse strawberry production. Some of the challenges include the initial cost of setting up the IoT equipment, energy consumption and adequate knowledge needed to install and use the devices. It is expected as IoT technology continues to grow, some of the challenges such as cost can be overcome.

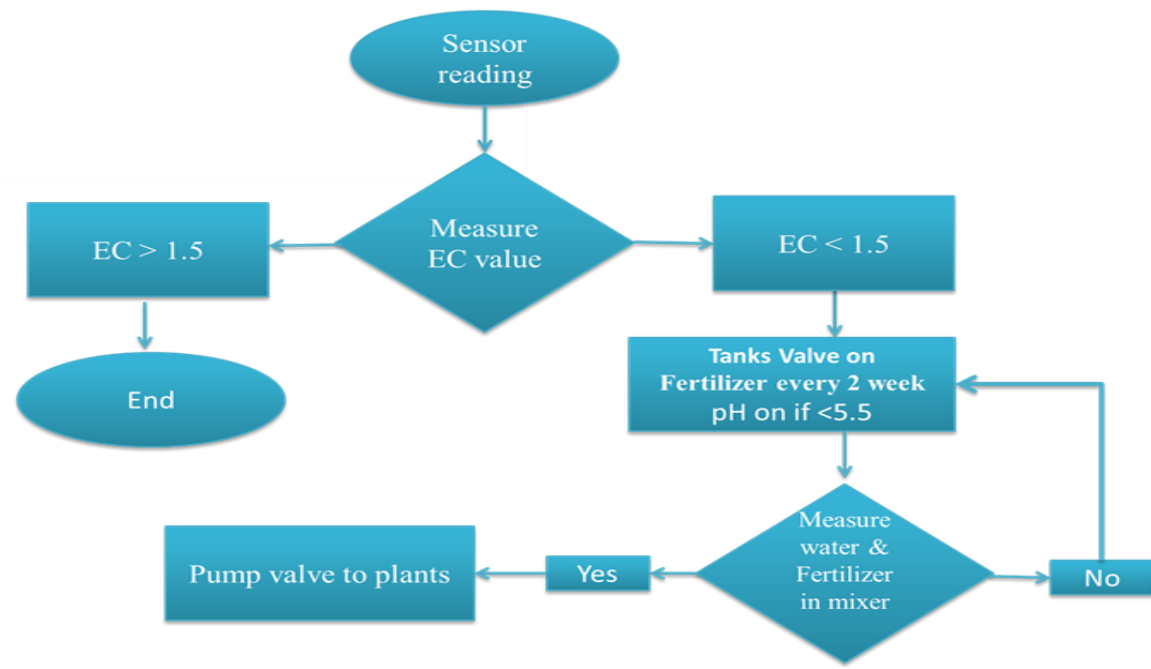

Figure 5. Typical flowcharts for automated system 


\section{FUTURE TRENDS}

The use of IoT has allowed for smart monitoring and control system in greenhouse strawberry. However, further research in IoT with data analytic and robotics can help study the optimal environmental conditions that can improve the quality of strawberry in greenhouse which are located in high temperate regions. In addition, preservation of strawberry using IoT needs to be further explored. The use of IoT can further be used in the harvesting and classification of strawberry in terms of size, sweetness, color and taste. The use of IoT technology to reduce energy consumption in greenhouse technology will attract much research interest. There is a need to explore low power wide range communication technology for greenhouse production especially in large farms with network limited coverage.

\section{CONCLUSION}

This paper presents a review of strawberry greenhouse based on IoT. Greenhouse production of strawberry allows availability of the fruit in season and off-season period during the year. The application of IoT in greenhouse provides several benefits such high efficiency, increase in productivity, reduction in labour, and ability to remotely control and monitor from anywhere and anytime. The use of low power wide area communication technology can help enhance the use of IoT in strawberry production. It will drive down energy consumption and cost of data transmission. The challenge associated with greenhouse production which include temperature, seasonal production and short shelf life can be overcome using IoT. The successful deployment of IoT greenhouse will allow for cultivation of strawberry in more locations in and across Malaysia.

\section{ACKNOWLEDGEMENTS}

The authors would like to express gratitude to the Ministry of Education (MOE) in Malaysia and Universiti Teknologi Malaysia (UTM) for providing the financial support for this research through the GUP grant (19H38) and HiCOE grant (4J216 and 4J209) which are managed by RMC UTM.

\section{REFERENCES}

[1] A. Kumar and I. Ahad, "Growth, yield and fruit quality of strawberry under protected cultivation in South Kashmir,” vol. 26, pp. 88-91, 2012.

[2] H. Kumakura and Y. Shishido, "Effects of temperature and light conditions on flower initiation and fruit development in strawberry," Jarq, vol. 29, pp. 241-250, 1995.

[3] J. Ruan, et al., "Flowering and fruiting of day-neutral and ever-bearing strawberry cultivars in high-elevation for summer and autumn fruit production in Korea," Hortic. Environ. Biotechnol., vol. 54, pp. 109-120, Apr 2013.

[4] A. B. U. B. Ibrahim, "Automated Temperature and Humidity Control System for Strawberry Plantation Using Solar Panel," pp. 14-19, 2015.

[5] "Season-Long Strawberry Production with everbearers."

[6] A. Sønsteby and O. M. Heide, "Temperature responses, flowering and fruit yield of the June-bearing strawberry cultivars Florence, Frida and Korona,” Sci. Hortic. (Amsterdam)., vol. 119, pp. 49-54, 2008.

[7] J. F. Hancock, et al., "Generating a Unique Germplasm Base for the Breeding of Day-neutral Strawberry Cultivars,” HortScience, vol. 53, pp. 1069-1071, Jul 2018.

[8] V. Winardiantika, et al., "Effects of high temperature on pollen performance in ever-bearing strawberry cultivars," Acta Hortic., vol. 1117, pp. 365-371, 2016.

[9] Q. Bai and C. Jin, "The Remote Monitoring System of Vegetable Greenhouse," 2017 10th Int. Symp. Comput. Intell. Des., pp. 64-67, 2017.

[10] D. M. Atia and H. T. El-madany, "Analysis and design of greenhouse temperature control using adaptive neurofuzzy inference system," J. Electr. Syst. Inf. Technol., 2016.

[11] R. Shahzadi, et al., "Internet of Things based Expert System for Smart Agriculture," Int. J. Adv. Comput. Sci. Appl., vol. 7, 2016.

[12] B. Zhang, et al., "Intelligent monitoring system of light intensity and CO2 concentration in strawberries greenhouse," 2017 IEEE Int. Conf. Mechatronics Autom., pp. 101-106, 2017.

[13] S. Kadir, et al., "Strawberry (Fragaria -ananassa Duch.) Growth and Productivity as Affected by Temperature," Hortscience, vol. 41, pp. 1423-1430, 2006.

[14] S. M. Zahedi and H. Sarikhani, "Effect of far-red light, temperature, and plant age on morphological changes and induction of flowering of a 'June-bearing' strawberry," Hortic. Environ. Biotechnol., vol. 57, pp. 340-347, 2016.

[15] Y. Li, et al., "Effect of LED supplemental illumination on the growth of strawberry plants," 2012 Symp. Photonics Optoelectron. SOPO 2012, 2012.

[16] E. Saenz, et al., "Strawberries collecting robot prototype in greenhouse hydroponic systems," Symp. Signals, Images Artif. Vis. - 2013, STSIVA 2013, 2013.

[17] Q. Feng, et al., "Study on strawberry robotic harvesting system," CSAE 2012 - Proceedings, 2012 IEEE Int. Conf. 
Comput. Sci. Autom. Eng., vol. 1, pp. 320-324, 2012.

[18] X. Li, et al., "A deep learning method for recognizing elevated mature strawberries," in 2018 33rd Youth Academic Annual Conference of Chinese Association of Automation (YAC), pp. 1072-1077, 2018.

[19] E. Avsar, et al., "Development of a cloud-based automatic irrigation system: A case study on strawberry cultivation," in 2018 7th International Conference on Modern Circuits and Systems Technologies (MOCAST), pp. $1-4,2018$.

[20] "IoT Based Greenhouse Monitoring using Data Compressive Sensing Protocol in WSN: a review," 2018.

[21] J. Li, "Design and Realization of Greenhouse Sensor Intelligent Management System Based on Internet of Things," Int. J. Online Eng., vol. 13, pp. 80, 2017.

[22] J. Zhao, et al., "The Study and Application of the loT Technology in Agriculture," Comput. Sci. Inf. Technol., pp. 462-465, 2010.

[23] R. Koshy, et al., "Greenhouse Monitoring and Control Based on IOT Using WSN,” pp. 59-64, 2016.

[24] S. Baker, et al., "Internet of Things for Smart Healthcare: Technologies, Challenges, and Opportunities," IEEE Access, pp. 1-1, 2017.

[25] S. R. Basavaraju, “Automatic Smart Parking System using Internet of Things (IoT),” Int. J. Sci. Res. Publ., vol. 5, pp. 629-632, 2015.

[26] T. Malche, "Internet of Things (IoT) for building Smart Home System," Int. Conf. I-SMAC, pp. 65-70, 2017.

[27] D. Zhang, et al., "The application of RFID-based on IOT in logistics management," Adv. Intell. Soft Comput., vol. 162 AISC, pp. 711-718, 2012.

[28] O. Elijah, et al., "An Overview of Internet of Things (IoT) and Data Analytics in Agriculture: Benefits and Challenges," IEEE Internet Things J., 2018.

[29] O. Elijah, et al., "Enabling smart agriculture in Nigeria: Application of IoT and data analytics," 2017 IEEE 3rd Int. Conf. Electro-Technology Natl. Dev. NIGERCON 2017, pp. 762-766, Jan 2018.

[30] G. N. Kodandaramaiah and V. Keerthi, "Cloud IoT Based Greenhouse Monitoring System," Keerthi.v Int. J. Eng. Res. Appl., vol. 5, pp. 35-41, 2015.

[31] R. S. Sinha, et al., "A survey on LPWA technology: LoRa and NB-IoT," ICT Express, vol. 3, pp. 14-21, 2017.

[32] U. Raza, et al., "Low Power Wide Area Networks: An Overview," IEEE Commun. Surv. Tutorials, vol. 19, pp. 855-873, 2017.

\section{BIOGRAPHIES OF AUTHORS}

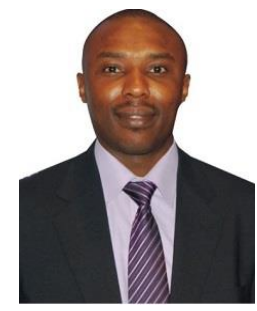

Olakunle Elijah received the B.Eng. degree from Federal University of Technology Minna, Minna, Nigeria, the M.Sc. degree in micro-electronics and computing from Bournemouth University, Poole, U.K., and the postgraduate certificate in advance microelectronics from Bolton University, Bolton, U.K., in 2003, 2008, and 2010, respectively. He received the Ph.D. degree at the Universiti Teknologi Malaysia, Johor Bahru, Malaysia, in 2018. He worked as a Field Engineer for Kuyet Nigeria Ltd., Lagos, Nigeria, in 2006. From 2011 to 2013, he was the MD/CEO at Microscale Embedded Limited, Abuja, Nigeria. His research interests include embedded systems, wireless communication, massive MIMO, radio frequency exposure for $5 \mathrm{G}$, and interference mitigation. He is the receipent of IEEE Malaysia ComSoc and VTS society joint chapter best paper award 2016. He is currently working as a research in the field of wireless communication with Wirless Communication Center.

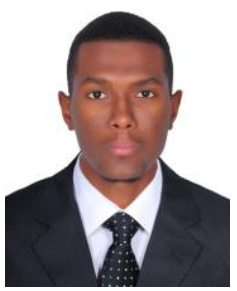

Abdelmoneim Mohamedosman received the B.E. of electronic and communication from Academy of engineering sciences, Sudan in 2015 and currently, he is a master student in Universiti Teknologi Malaysia Johor Bahru, Department of Electronic and Telecommunication. He worked as a teacher assistant from 2015 to 2016 at (Academy of engineering sciences, AlMughtaribeen and Razi) University. His research interests include network routing algorithms, wireless sensor networks, antenna propagation, IoT, 5G communications, and reliability of the novel computer architecture such as embedded system.

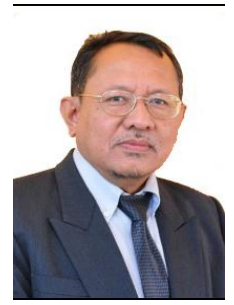

Prof. Dr. Tharek Abd Rahman is a Professor at Faculty of Electrical Engineering, Universiti Teknologi Malaysia (UTM). He obtained his BSc. In Electrical \& Electronic Engineering from University of Strathclyde UK in 1979, MSc in Communication Engineering from UMIST Manchester UK and PhD in Mobile Radio Communication Engineering from University of Bristol, UK in 1988. He is the Director of Wireless Communication Centre (WCC), UTM. His research interests are radio propagation, antenna and RF design and indoors and outdoors wireless communication. He has also conducted various short courses related to mobile and satellite communication to the Telecommunication Industry and government body since 1990 . $\mathrm{He}$ has a teaching experience in the area of mobile radio, wireless communication system and satellite communication. He has published more than 250 papers related to wireless 
communication in national/international journal and conference.

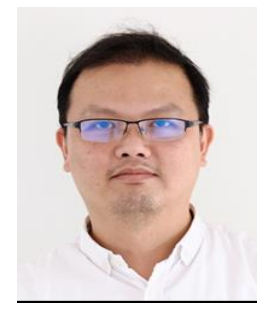

Tien Han Chua received both the B.Sc. (Honours) degree in Electrical engineering (First Class) and the Master of Electrical Engineering in Wireless Engineering from the Universiti Teknologi Malaysia in 2003 and 2007, respectively. Tien Han was a Tutor (2005-2007) and then a Lecturer (2007-present) at the Faculty of Electrical Engineering, Universiti Teknologi Malaysia.

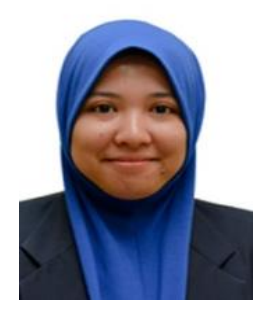

Siti Fatimah AUSORDIN received her B.Eng. (Telecommunication) and M.Eng from Universiti Teknologi Malaysia, in 2010 and 2013 respectively. Her research areas include microwave devices and butler matrix beam forming system. She is now currently working as a reseaech officer with Wireless Communication Center, Universiti Teknologi Malaysia.

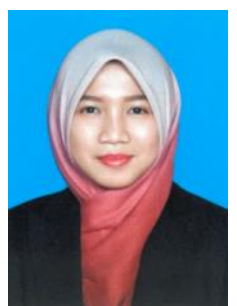

Rifhan Narrissa binti Razali holds a Bachelor Degree of Electrical and Electronics Engineering from Universiti Putra Malaysia (UPM) in 2016. She currently works in Wireless Communication Centre (WCC), Universiti Teknologi Malaysia (UTM) as an Assistant Research Officer. She has worked as Application and Customer Support Engineer at Controls Handling Engineering Sdn Bhd and received training as IT Support Engineer at Nityo ICoE Sdn Bhd. 